\title{
COMPARAISON DE DIVERSES MÉTHODES DE CALCUL APPLIQUÉES A LA CHAMBRE D'ÉQUILIBRE COMPLEXE DE L'USINE DE BIOGE (HAUTE-SAVOIE)
}

par L. ESCANDE, Ingénieur I.E.T.

Professeur à la Faculté des Sciences de Toulouse

Directeur de l'Institut Electrotechnique et de l'Institut de Mécanique des Fluides

et

V. GOUTKIN, Ingénieur I.E.G. et E.I.H.

\section{I. - DONNÉES ET ÉTUDES PRÉLIMINAIRES}

L'usine de Bioge, à qüelques kilomètres de la ville de Thonon-les-Bains, est actuellement alimentée par les eaux de la Dranse-d'Abondance, dérivées dans un souterrain en charge de $2 \mathrm{~km} .2$ environ de longueur.

Pour le compte d'Electricité de France, Maître de l'CEuvre, des travaux sont actuellement en cours, qui ont pour but de doubler la puissance installée à l'usine en utilisant également les eaux de la Dranse-de-Morzine, de sorte que l'ensemble de l'aménagement constitue une chute en $Y$ dont les organes communs seront essentiellement la chambre d'équilibre et les conduites forcées. Le débit maximum dérivé passera ainsi de 10 à $20 \mathrm{~m}^{3} / \mathrm{sec}$.

La chambre d'équilibre existante, aménagée à l'extrémité aval de la galerie d'amenée d'Abondance, est du type classique à double épanouissement. Insuffisante pour répondre aux besoins hydrauliques du nouvel aménagement, il a fallu songer d̀ l'agrandir, en tenant compte toutefois du fait qu'elle est construite dans un terrain dont la médiocrité a nécessité de solides revêtements en béton armé.

On s'est donc astreint à modifier le moins possible l'ouvrage existant, en le doublant par un ouvrage similaire mais de réalisation presque entièrement indépendante. C'est ce qui explique la complexité apparente des dispositions prévues (fig. 1).

Du point de vue fonctionnel ces deux ouvrages se confondent.

En effet, la proximité relative des puits distants d'une cinquantaine de mètres et leur large intercommunication réalisée par l'épa-

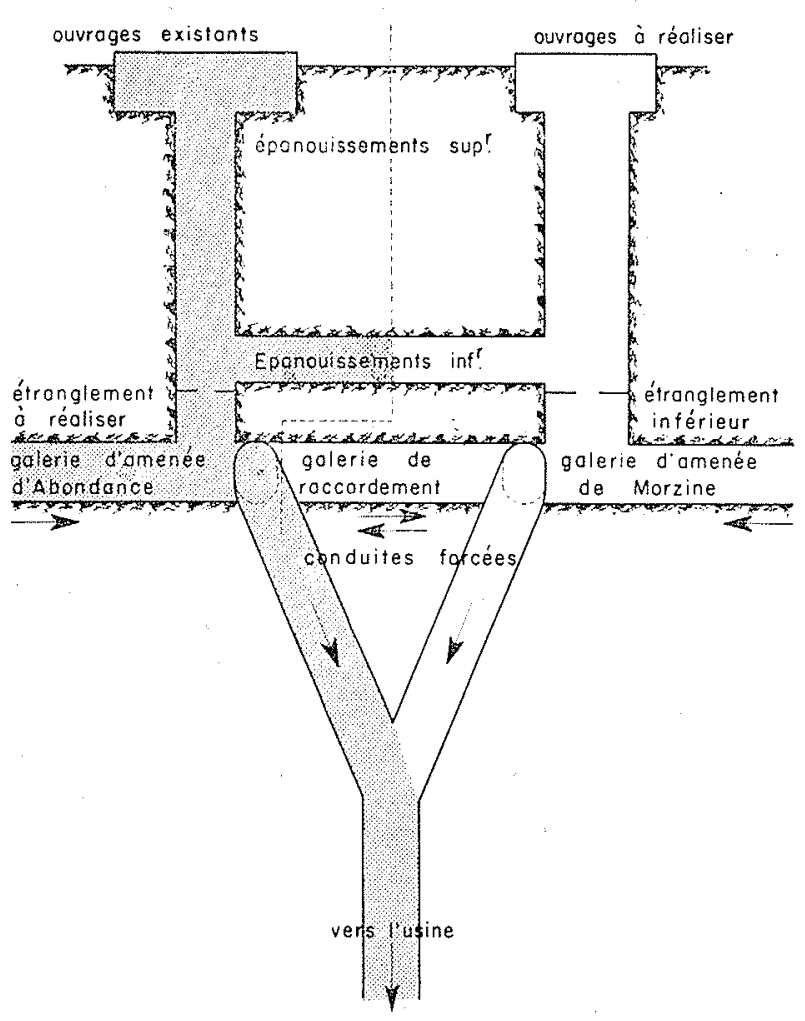

fig. 1

nouissement inférieur, assurent constamment, à la valeur négligeable de la perte de charge près, dans la galerie de raccordement, une égalisation des niveaux piézométriques dans les deux chambres.

Nous pouvons donc assimiler cet ensemble à une seule chambre d'équilibre, du type à épanouissement supérieur et inférieur, et munie en outre d'un étranglement inférieur. 
Ce premier point étant établi, il reste à dimensionner cette chambre dont la particularité est d'être alimentée par 2 galeries d'amenées divergentes, puisant dans les retenues d'Abondance et de Morzine dont les niveaux statiques peuvent varier considérablement sans aucune relation mutuelle, respectivement de 647,50 à 643,50 et de 647,50 à 639,00 (fig. 2).

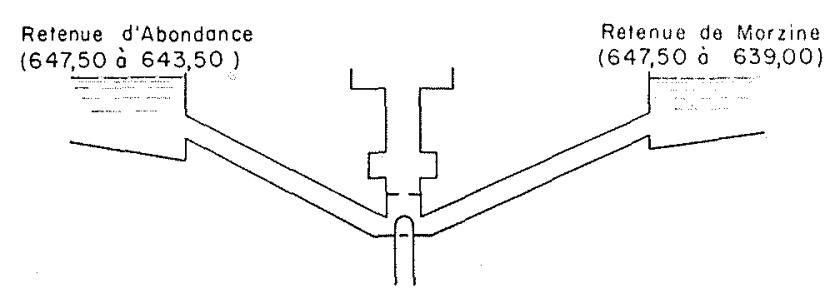

fig. 2

Les niveaux statiques considérés, pour l'étude du phénomène consécutif à un arrêt du débit de I'usine, ont été évidemment pris égaux à leur valeur maximum commune soit à la cote 647,50 . Par contre, il a fallu procéder par quelques tâtonnements à la détermination des 2 niveaux les plus défavorables, dans le cas d'un démarrage brusque de la moitié de lá puissance totale de l'usine, condition imposée par les services d'exploitation et équivalent à l'ouverture brusque du débit de 10 à $20 \mathrm{~m}^{3} / \mathrm{sec}$.

L'application de la méthode aux différences finies (1) eut été dans ce cas fort laborieuse. Par contre, la méthode graphique exposée dans un article précédent (2) nous a permis d'arriver très rapidement au résultat.

Cing cas différents ont été examinés, qui nous ont permis de conclure que les niveaux les plus dévorables sont :

643,50 pour Abondance.

639,00 pour Morzine.

Ce résultat n'était pas évident à priori car, pour un même débit total utilisé, si le débit et par conséquent la perte de charge diminuent dans la galerie alimentée par le lac inférieur à mesure que croît la dénivellation entre les deux retenues, ils augmentent par contre dans l'autre galerie.

Des caractéristiques de l'aménagement étudié, il ressort que l'effet d'accélération des masses, dû à la différence existant à chaque instant entre le niveau statique de la retenue la plus haute et le niveau du plan d'eau dans la chambre, l'emporte sur l'effet de freinage dû à la perte de charge dans la galerie correspon- dante. Mais rien ne permet d'affirmer que dans $d^{\prime}$ autres aménagements similaires, il en sera toujours ainsi.

\section{II. - ÉTUDE PROPREMENT DITE DE LA CHAMBRE D'ÉQUILIBRE}

\section{a) Condition d'amortissement des oscillations (formule de THOMA).}

Une étude exacte de la question nous conduirait à des calculs inextricables. Ce n'est que dans I'hypothèse des lacs au même niveau que I'on arrive aisément à la solution.

En effet, en reprenant dans ce cas, la "formule de THOMA» sous sa forme habituelle:

$$
\text { Fo }>\frac{Q_{0}{ }^{2}}{2 g \text { PwO Ho }} \frac{L}{f}
$$

dans laquelle:

Pwo représente la somme des pertes de charge dans l'une ou l'autre des 2 galeries d'amenée.

$\mathrm{Ho}_{\mathrm{O}}=\mathrm{H}$ - Pwo est la chute mesurée entre le niveau piézométrique dans la chambre et le niveau de restitution aux turbines.

L

- le rapport de la longueur à la section du canal équivalent, et en remarquant que (3) :

$$
\frac{L}{f}=\frac{1}{\frac{f 1}{L 1}+\frac{f 2}{L 2}}
$$

on a immédiatement :

$$
\text { Fo } \frac{Q_{0}{ }^{2}}{2 g \text { Pwo Ho }} \frac{1}{\frac{f 1}{L 1}+\frac{f 2}{L 2}}
$$

On pourrait évidemment pousser plus loin la précision en tenant compte de l'influence de la hauteur représentative de la vitesse d'écoulement dans les galeries au point d'insertion de la chambre d'équilibre (4).

Cette hauteur se détermine, ici, non par la Wo'

formule $\frac{}{2 g}$ utilisée dans le cas d'une galerie 
d'amenée unique, mais par application du théorème des forces vives aux masses d'eau au voisinage de la chambre d'équilibre.

Elle est ainsi :

$$
P^{\prime \prime} 0=\frac{1}{2 g} \frac{\left(W_{1}^{\prime} q_{1}+W_{2}: q_{2}\right)}{Q_{0}}
$$

Sans entrer dans le développement des calculs, nous nous contenterons de donner, ici, le résultat auquel on aboutit :

$$
\text { Fo }>\frac{Q_{0}{ }^{2}}{2 g P_{w o}\left(H_{0}+2 p^{\prime \prime} \mathrm{o}\right)}-\frac{1}{\frac{f 1}{L 1}+\frac{f 2}{L 2}}
$$

avec $\mathrm{Ho}_{\mathrm{O}}=\mathrm{H}-\mathrm{Pwo}+\mathrm{P}^{\prime \prime} \mathrm{o}$

La considération du terme $P^{\prime \prime}$ o permet donc théoriquement de réduire la section minimum Fo de la chambre.

Mais outre que cette réduction est en général à peine sensible, vu la faible valeur de $\mathrm{P}^{\prime \prime} \mathrm{O}$ relativement à la chute, la marge de sécurité que l'on a coutume de s'imposer entre la valeur minimum de Fo déterminée par le calcul et celle admise en pratique, rend illusoire le bénéfice qui résulte de l'utilisation de cette dernière formule. Son application s'impose néanmoins dans le cas où l'on veut remédier à une instabilité reconnue des groupes, en provoquant de grandes vitesses au droit de la chambre.

b) Valeur nécessaire Ro de la perte de charge produite par l'étranglement inférieur traversé par le débit total aménagé de $20 \mathrm{~m}^{3} / \mathrm{sec}$.

Nous avons été guidés dans le choix de cette valeur par les deux conditions suivantes:

- obtenir, lors de l'ouverture la plus dangereuse, une dépression maximum correspondant à la plus forte descente de l'oscillation.

- lors de la mancuvre la plus défavorable de fermeture, limiter la montée maximum de l'oscillation à une cote inférieure à 650.76, niveau d'arasement de l'ouvrage existant.

Quelques essais nous ont fait adopter, pour Ro, la valeur optimum de 12 mètres.

c) Etude des phénomènes consécutifs à des manœurres de fermeture totale.

Les 2 lacs étant tous deux à leur niveau maximum 647.50, cette étude n'a présenté aucune difficulté.

L'application successive des méthodes gra- phique et analytique nous a conduits aux

\begin{tabular}{|c|c|c|c|}
\hline \multirow{3}{*}{$\begin{array}{l}\text { Manœuvre } \\
\text { de fermeture } \\
\text { tofale }\end{array}$} & \multirow{2}{*}{\multicolumn{2}{|c|}{$\begin{array}{c}\text { Montée maximum } \\
\text { de l'oscillation } \\
\text { METHODE }\end{array}$}} & \multirow{3}{*}{$\begin{array}{l}\text { Cote } \\
\text { correspondante } \\
\text { à la surpression } \\
\text { maximum }\end{array}$} \\
\hline & & & \\
\hline & ohiaue & Analyt & \\
\hline nsta & 650.60 & 650.53 & 657.22 \\
\hline $40 \mathrm{sec}$ & 650.65 & & 653.57 \\
\hline
\end{tabular}
résultats suivants :

d) Manœuvre instantanée d'ouverture de 10 à $20 \mathrm{~m}^{3} / \mathrm{sec}$.

La méthode graphique conduit à une dépression maximum de $10 \mathrm{~m} .42$ par rapport au niveau statique 643.50 du lac le plus élevé, et à une descente maximum du plan d'eau égal à $10 \mathrm{~m} .30$

En traitant le même problème par la méthode aux différences finies, on obtient une descente maximum de $10 \mathrm{~m} .52$ et une dépression maximum de $10 \mathrm{~m} .48$

En réalité, la chambre d'équilibre de Bioge présente un épanouissement inférieur de sections horizontales variables, auxquelles nous avons substitué une section constante qu'on pourrait désigner par section moyenne pondérée, chaque section devant être affectée d'un coefficient d'autant plus fort qu'elle est plus basse.

L'étude précise du phénomène, en tenant compte de la variation des sections, ne peut rationnellement se faire que par la méthode aux différences finies, car la méthode graphique implique nécessairement une section constante sur un minimum de hauteur compatible avec un tracé d'épure.

En faisant intervenir à chaque instant la valeur effective de la section considérée, en fonction de la cote du plan d'eau dans la chambre, la méthode D.F. conduit à une descente maximum de $10 \mathrm{~m} .36$ et à une dépression maximum de $10 \mathrm{~m}$. 30 , chiffres pratiquement égaux aux précédents.

\section{e) Conclusion.}

On constate ainsi que les résultats fournis par la méthode graphique (2) ne diffèrent de ceux obtenus à l'aide des méthodes analytiques ou aux différences finies (1) que d'environ $2 \%$ au maximum.

Compte tenu du degré d'exactitude des épures et de l'approximation des calculs, on peut admettre que cette méthode graphique constitue, en raison de sa simplicité, un procédé de calcul précis et particulièrement rapide. 


\section{f) Remarque.}

En raison des phénomènes de balancement susceptibles de se produire entre les 2 chambres d'équilibre, phénomènes qui ne sauraient avoir d'ailleurs qu'une amplitude limitée, il nous a paru nécessaire de prévoir une certaine marge de sécurité en aménageant un léger épanouis sement au sommet du nouveau puits.

ANNEXE

PRINCIPES

DE LA MÉTHODE AUX DIFFÉRENCES FINIES APPLICABLE

LORS D'UNE OUVERTURE INSTANTANÉE,

DANS LE CAS

D'UNE CHAMBRE A SECTION VARIABLE ALIMENTÉE PAR 2 LACS

DONT LES NIVEAUX SONT DIFFÉRENTS :

Les équations générales donnent, avec les notations habituelles, les relations suivantes, valables durant toute la première descente du plan d'eau dans la chambre:

$$
\begin{gathered}
V=\frac{1}{F}\left(f_{1} w_{1}-f_{2} w_{2}-Q_{11}\right) \\
\Delta W_{1}=\frac{-g}{L I}\left(Z_{1} K_{1} W_{1}^{2}-\lambda F^{2} V^{2}\right) \Delta t
\end{gathered}
$$

$$
\begin{gathered}
\Delta W_{2}=\frac{-g}{L 2}\left(Z_{2}+A+K_{2}\right. \\
\left.W_{2}^{2}-\lambda F^{2} V^{2}\right) \Delta t \\
\Delta Z=\frac{F V \Delta t}{F+\frac{\Delta F}{2}}
\end{gathered}
$$

Le terme (A) de la relation [3] désignant la différence de niveau entre les deux lacs.

On applique tout d'abord les relations [1], $[2],[3]$, en admettant pour $F$ la section correspondant à la cote initiale $Z$, à l'instant $t=0$.

La relation [4] se résoud par approximations successives en prenant:

$$
F=0 \text { d'où } \Delta Z_{1}=V \Delta t \text { et une }
$$
première valeur $\Delta F_{1}$

$$
\Delta F=\Delta F_{1} \text { d'où } \Delta Z_{2}=\frac{F V \Delta t}{F+\frac{\Delta F_{1}}{2}} \text { et }
$$

une deuxième valeur $\triangle F_{2 .}$ etc... jusqu'à obtenir :

$$
\Delta F_{n}=\Delta F_{n+1}=\Delta F
$$

et par conséquent :

$$
\Delta Z_{n}=\Delta Z
$$

On retient alors pour l'intervalle suivant les valeurs:

$$
F+\triangle F \text { et } Z+\triangle Z
$$

(1) L. ESCANDE : "Cénie Civil " des 15 juillet et 15 octobre 1946.

(2) V. GOUTKIN: "Méthode générale de calcul des chambres d'équilibre alimentées par 2 canaux d'amenée.

(3) L. ESCANDE: "Génie Civil " des lor et 15 avril 1943.

(4) CALAME et CADEN: "Schweizerische Bauzeitung" du 